ment followed in the German case, and the total neglect of thoracentesis, by one of the most eminent clinical teachers of his time, be the usual course, likely to be followed by his pupils now spread over Germany, I think the opinion I shall express, and the advice I shall give, proper and just. If, moreover, it be a fact that thoracentesis, as performed upon the European continent, has proved fatal in many instances, whereas I have yet to learn of any such fatality in New England, and, I think, in America, and when I myself have never met with a deatb, I am persuaded that the operator is in fault in Europe. I believe the operation, if carefully done, with due regard to the first moment of suffering on the part of the patient (cough, dyspnœea, stricture of chest), and if immediately thereupon the trocar be removed, is as simple and innocuous as venesection or vaccination. Such a remark does not apply to the operation, as performed in Europe.

My advice, therefore, to my patient visiting Europe will be as follows: If you find yourself threatened with pleurisy, send for an American physician, and trust to him rather than to any other under the influence of continental therapeutics. One exception I feel bound to make. If yon are in Paris, summon Mons. Dieulafoy, immediately. He does not decline thoracentesis when necessary; and his rules for the operation are perfectly safe.

This may seem gross presumption on my part, nevertheless I shall feel it my duty to give the advice, upon the argument that, at times, much suffering may be prevented and, possibly, some lives may be saved.

\section{CASES OF CONTRACTION AT THE HIP-JOINT.}

BY E. H. BRADFORD, M, D.

It has been abundantly proved in surgical practice that obstinate flexion of the femur can be corrected by osteotomy, by osteoclasis, by brisement force, in certain cases, by tenotomy followed by gradual or immediate straightening. ${ }^{2}$

It is also known that in the early stage of hip disease, before the tissues have become contracted, simple rest in bed will allow the limb to correct itself, or, under an anæsthetic the limb can easily be placed in position. ${ }^{3}$

After the disease has lasted for some time a contraction, which has been aptly termed " adapted shortening," takes place, and under an anæsthetic the limb cannot be replaced by the employment of force alone. In one cadaver on which I had an opportunity of examining a contraction of this sort, perfect correction could not be gained (although no bony union or firm fibrous adhesions were present) even after the skin was torn and some of the muscles divided.

As is well known, the acetabulum becomes widened and the head of the femur absorbed under the reflex muscular pressure which crowds the femur backward and upward, there being no muscle to pull the limb downward. This produces a position of pseudo dislocation, which, with contraction of the adjacent tissues, constitutes the deformity.

In the following cases treatment was attempted without strictly operative intervention :-

1 Read before the Boston Society for Medical Improyement, January $9,1882$.

2 Buckminster Brown. The Journal, June 2, 1881.

8 Case II., Journal, November 11, 1880, page 467 .
Case I. R. W., six years old, of healthy parentage, and without a history of previous illness. There is no phthisis in the family. The boy met with a slight fall, previous to the attack of hip disease. This was first noticed in the summer of 1880 , and for this he was treated by different regular physicians, for a while in the autumn of 1880 was placed for three months under the care of the Italian bone-setter, Regina Dal-Cein; by her his limb was poulticed and pulled by the hand. He is thought by the mother to have improved while under this treatment, the limb which was somewhat flexed becoming less so. A few months later, however, two abscesses formed; these were incised by the family physician, and after discharging for several months the sinuses healed. The limb began, however, to "draw up," and on September 6th the thigh was flexed at a right angle to the axis of the sacrum. There was no swelling or tenderness about the hip. The head of the trochanter was not above the Nélaton line, and any attempt to extend the thigh caused arching of the lumbar spine. 'The patient suffered no pain, and the limb was but slightly adducted.

With the assistance of Dr. Otis the boy was etherized, and the limb forcibly pulled. Tenotomy was not done. Comparatively little was gained in this way. An extension splint was then applied, the leg being kept elevated from the plane of the bed to an amount nearly equal to the degree of deformity, A folded pillow was placed under the knee, and an extension by weight and pulley (seven pounds) attached to the end of the splint.

The patient was quite restless the night following the operation, and complained of some pain referred to the knee, and slight muscular twitching was noticed if the extension splint was removed, followed by pain referred to knee. 'This lasted, however, but about twenty-four hours. At the end of three days the limb was within twenty degrees of the axis of the body. At the end of a week the leg had been brought to the line of the axis of the body. He was allowed to get

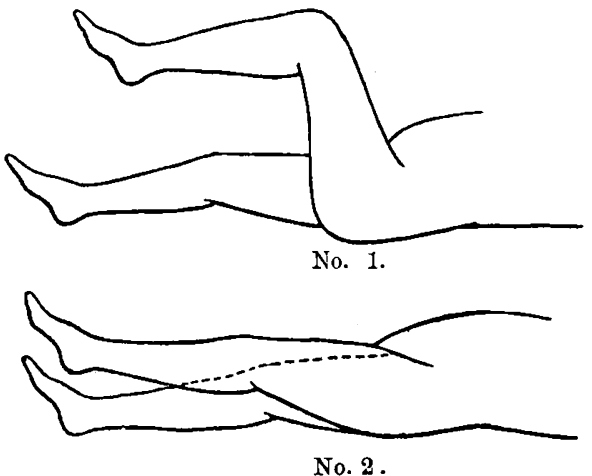

up and go about with crutches, and elevated shoe applied to the well foot, wearing the extension splint on the affected limb. No swelling of the hip, tenderness, heat, or redness followed, and the boy remained in every way as well as before the stretching.

At the present time, four months later, there is complete absence of any deformity; there is no difference in the length of the limbs; the boy is able to stand on the affected limb, but as a protection against jar or possible recurrence of the deformity is directed to wear an extension splint.

The condition before operation is accurately indicated in the accompanying outline sketch, No. 1, and 
in No. 2 the position of the limb after two and three weeks' treatment.

Case II. R. F., aged seven, patient at the City Hospital ; history that of hip disease of one and a half years' duration. At the time of entrance there was no tenderness about the hip, no motion at the hip. The thigh was flexed to nearly a right angle, and slightly adducted.

The same treatment was carried out in this case as in the previous one except that no extension splint was used. A ham splint was bandaged to the limb to prevent flexion of the femur, and a weight and pulley applied. After etherization the limb could not be straightened more than through an arc of forty degrees. Extension was therefore applied with the leg raised to that amount. This was gradually reduced. At the end of a fortnight extension was in the line of the bed on which the child lay. A certain amount of sagging of the bed prevented perfect correction of the deformity, and the child's hips were therefore raised by placing a pillow under the buttocks. This raised the pelvis slightly above the line of the extension.

At the end of eight weeks he was furnished with crutches and an elevated shoe, and wearing the ham splint allowed to walk about. A week later he was allowed to go about on crutches without the ham splint, and no evil results followed the forcible manipulation.

The skin near the anterior superior spinous processes was slightly torn at the time of the straightening, but no inflammation followed.

The correction of the deformity followed in this, as in the previous case, without difficulty. At the time of discharge from the hospital the patient's limbs were nearly parallel, the affected limb being flexed at the femur at an angle of ten degrees with the axis of the sacrum. After discharge from the hospital careful treatment was impossible.

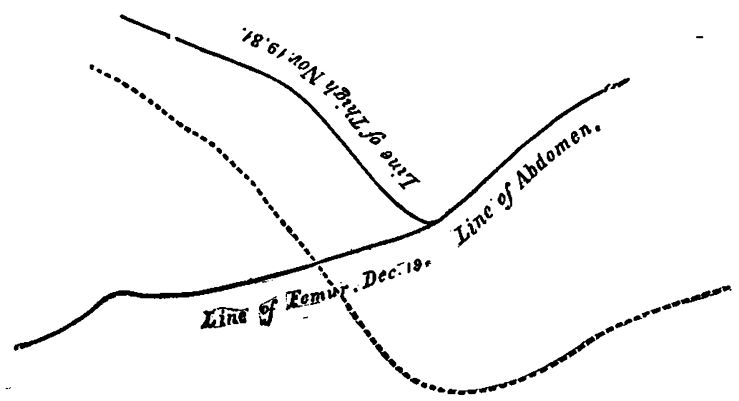

No. 3.

Case III. J. S., aged three, hip disease of one year's duration. The patient entered the hospital with the femur flexed firmly, nearly at a right angle with the axis of the trunk, and slightly adducted. There was no tenderness at the hip. The child was a delicate one, but was fairly nourished.

Extension by weight and pulley (seven pounds) was applied in nearly the line of the deformity. To accomplish this a cross-bar was fixed over the foot of the bed and several feet above it. A pulley was fastened to this, over which ran the cord for the ordinary weight and pulley extension. A board covered by a pillow was placed beneath the limb, extending from the buttock to the pulley. The knee was bandaged to this, and the limb in this way supported in the line of the deformity. The board and pulley were lowered from day to day as far as was possible without tilting the pelvis. At the end of three weeks the deformity was so far corrected that the pelvis did not tilt when the limb was placed upon the level of the bed. The skin in the groin gave evidence of a great deal of stretching, and the patient suffered somewhat from nocturnal pain for ten days, but no disturbance of the general condition followed, and the patient is at present undergoing treatment. The amount of deformity and of correction is indicated by the accompanying tracing, No. 3, taken by placing a strip of lead upon the abdomen and anterior surface of the femur.

In these three cases the deformity was of an aggravated type. In two under an anæsthetic but' little was gained except after traction. In the third the weight of the limb was utilized in addition to traction as a means of completing the rectification of the deformity. The amount of force which can be used in this way is considerable, as can be easily demonstrated if any one stands or lies with the limb held for a time at a marked angle with the body (the leg being kept extended and not flexed at the knee). The amount varies with the length and size of the limb.

The accompanying case, an aggravated one, of many years' standing, with cicatrices of old sinuses, shows that something may be gained by this means alone.

CASE IV. E. A., a young woman twenty-two years old. The patient first suffered from hip disease when three years old; her trouble was of an aggravated type. Abscesses formed and sinuses continued to discharge for many years.

At the time of commencement of treatment her thigh was flexed at an angle of about $70 \circ$ with the axis of the sacrum. The arm was somewhat adducted. The trochanter was two inches above the line passing between the anterior superior spine and the tuberosity of the ischium. The cicatrices of two sinuses were present about the hip and no motion was to be detected. 'The patient used on this limb a shoe raised five inches, and with this, with difficulty, touched the floor with her heel. The deformity was so marked that it did not appear to be at all probable that any benefit could be derived from non-operative treatment, and nothing would have been attempted, if Dr. C. P. Putnam had not felt confident that the conditions could be improved.

The patient was placed in bed and a ham splint applied to prevent flexion of the knee. This was secured by means of adhesive plaster and a silicate bandage. After the patient had become accustomed to this position, she, a few days later, was allowed to go about as freely as she desired, using crutches. No traction or friction was used, and the correction of the deformity was left entirely to the weight of the limb. This power of course only acted while the patient was standing, and necessarily a great deal of the time the limb was without any correcting force.

At the end of four months a removable splint was applied instead of the ham splint, answering the same purpose, namely, prevention of flexion at the knee.

At the end of six months the deformity had been overcome to an extent indicated by the accompanying tracings, ${ }^{1}$ No. 4 and No. 5, and the patient is able to touch her heel to the ground with a shoe elevated three inches. She has during the treatment suffered from a slight amount of transitory pain. At the present time she is

1 These tracings were taken by placing the patient upon a large sheet of stout paper, and with a pencil marking the outline of the body and limb. They were reduced by photography to their present size. No. 4 shows the patient before, No. 5 after treatment. 
able to walk about, using one crutch to steady herself, but is able to bear full weight upon the affected limb.

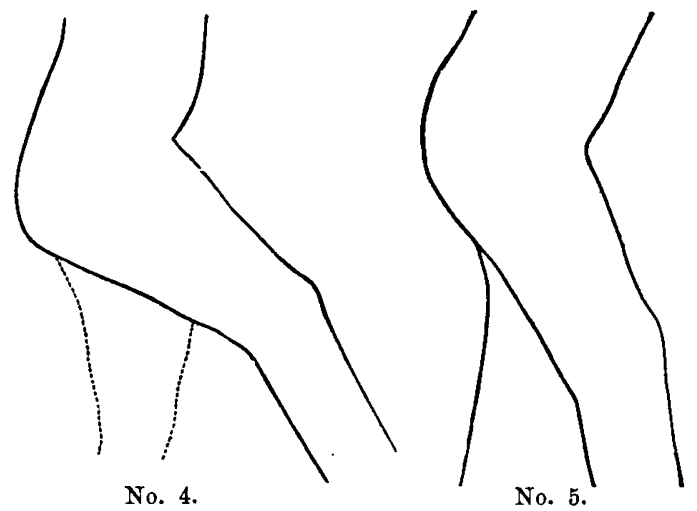

In cases where the deformity has not been of long standing, even of a pronounced type, a light extension applied without regard to the line of deformity is sufficient to gain the result.

CASE V. M. C., aged eight years ; hip disease of six months' duration. 'The patient's limb was flexed at a right angle with the line of the body. 'The patient had but little pain or tenderness. A light extension by weight and pulley was applied, the patient being fixed in bed, and the limb in two weeks became perfectly straight. Several months later an abscess formed and in a year the patient died with disease of the liver and kidney.

Case VI. M., patient aged five, hip disease of four months' duration; Hexion of the left thigh to $40^{\circ}$. The child was confined and fixed in bed, a light extension by weight and pulley applied without regard to the direction of the deformity; in two weeks the limbs were perfectly parallel and no deformity remained. The patient was kept in bed for several months. At the present time, six months later, tbe child is allowed to walk about, wearing an extension splint.

The following is a case of deformity of hip disease complicated by actual dislocation, as was discovered on etherization : -

Case VII. C. W., five years old, a patient at the Children's Hospital, was admitted suffering from hip disease of right side of eighteen months' duration, with an acute exacerbation. The limb was extremely tender, was flexed and adducted. The hip was swollen, hot, and tender. The patient was etherized, and in extending the limb in the manipulation for correction of the deformity the head of the femur was felt to slip into the acetabulum. No bony grating was detected. The correction of the deformity was not complete, as some flexion remained. An extension of eight pounds in two weeks' time brought the limb to the normal position. The patient was relieved of pain and improved in general condition. An abscess, however, formed three montbs later, and shortly afterwards the child was attacked by diphtheria and died.

As is well known, deformities at the hip joint can in many cases be overcome by the methods of securing by bandages the patient to a frame which embraces the trunk and extends along the affected limb. The frame at first is bent according to the amount of distortion, but it is gradually straightened and the limb bandaged in the straightened position.

The following case, one of periarticular disease, illustrates this method:-
Case VIII. Agnes Powers, a child three years old, entered the Children's Hospital with a right angled contraction at the left hip. Tenderness over the sacro-iliac synchondrosis was marked, and the usual symptoms of hip disease being absent a diagnosis of sacro-iliac disease suggested itself. An abscess, however, formed and on incision no diseased bone could be detected, and the incision healed after thorough drainage. The patient was placed upon a wire frame which reached from the axilla to below the left foot, this was bent according to the amount of flexion, but was straightened from day to day. At the eud of three weeks the limb was entirely straight.

It would be too much to assume that the correction of a deformity of this sort was the cure of it. As long as any inflammation remains at the hip joint, or as long as from timidity or habit of disuse the patient is accustomed to hold the limb in an unnatural position, a recurrence of the deformity, to a certain degree, may be considered possible or probable.

To prevent this recurrence either the continued use for a longer or shorter time of the measures taken for the correction of the deformity is necessary, or the treatment of the latent disease at the joint, a description of which does not fall within the scope of this paper, the object of which is to indicate that in certain cases of this class of deformity correction can take place without the usually mentioned methods of procedure.

\section{PARAPLEGIA (PARAPARESIS) FROM FUNC- TIUNAL WEAKNESS. ${ }^{1}$}

BY S. G. WEBBER, M. D.

I wILL describe three cases which illustrate three varieties of the affection to which $I$ wish to call attention.

M. J. F., widow, aged twenty-six, entered the City Hospital August 27, 1879. Diagnosis endocervicitis. She had a miscarriage eight years before, followed by inflammation of the womb; this seems to have been unimportant, as she soon recovered from it, and was transferred to nervous and renal service September 24 th.

The patient had never been strong; had had all the usual diseases of childhood, lung fever two or three times, typhus fever when a child, rheumatic fever nine years ago, and intermittent fever, typhoid fever twice, the last time March, 1878, complicated with spinal meningitis. When thirteen years old she fell on the ice upon her spine; again, at fifteen years of age, fell down a flight of stone steps, striking upon lower part of spine; was laid up by this for three months, having great difficulty in walking and sitting. After the typhoid fever and spinal meningitis in 1878 she was confined to the house till January 1, 1879. She had had pain in her back for a year, pain in right leg, more than in left, for a year; pricking sensations in legs with numbness for a year past; had had a sense of a girdle about waist; some para-æsthesia in legs and back, hyperæsthesia of back, the weight of the bed-clothes lying on her back causing pain; micturition rather difficult, sometimes only once in forty-eight hours; vision defective; diplopia; tremor of hands during voluntary action for the past six months.

1 Read before Boston Society for Medical Improvement, January 9,1882 . 